\title{
A classification of clinical paediatric research with analysis of related ethical themes
}

\author{
John Pearn University of Queensland, Australia
}

\section{Author's abstract}

Different types of clinical research are associated with different degrees of risk and with varying utility. Usually classified as therapeutic or non-therapeutic, clinical research involving children necessitates a balance between the conflicts of intrusion into a group of vulnerable subjects, and the obvious advantages which such intrusion engenders. To understand better the potential ethical dilemmas of paediatric research the author has expanded the classification of such clinical research involving children. Five types of such research - preventive research, curative research, research to alleviate symptoms, studies to establish norms and baselines, and curiosity researchare discussed in the context of their ethical constraints, and the different ethical questions which confront workers operating in each of these different themes.

\section{Introduction}

Clinical research involving children is necessary for the continued betterment of children's welfare. Doctors who work with children have a duty to investigate possibilities of cure, or the alleviation of symptoms. This subject is a topical one both in Britain $(1,2)$ and in the United States (3-8), and in other Western countries (9-11). Clinical research demands a compromise between essential and necessary advances in medical knowledge on the one hand, and between interference with and possible exposure to risk or discomfort (12), on the other. It is important that all who work with children are aware of the broader consequences of any non-therapeutic intervention into the lives of children (13), and of the special place of the child who is the subject of a therapeutic research endeavour (2).

There exists a fundamental moral dispute whether children should be used at all in research which is not designed (or is unlikely) to benefit them as individuals. In one sense children are no more vulnerable than are adults who may be subject to research overtures the details and ramifications of which they cannot

\section{Key words}

Ethics; paediatric research; classification; non-therapeutic research; research risks; institutional ethics committees. interpret. On the other hand, the use of children as a means to further individual research careers per se is repugnant to most. Research involving children may be classified as therapeutic or non-therapeutic (11), or more simply as research which involves different categories of risk. It is generally accepted that children should not be involved in non-therapeutic research if there is any risk, and moral safeguards are mostly in place to prevent this possibility. However, utility and benefit on the one hand and risks and dangers on the other are concepts of relativity varying along a continuum.

One approach to decision-making involving the allowability of child-based research is to consider specific cases within the classificatory framework. Research which uses children as subjects can be divided into a number of different qualitative types. It may be helpful for a potential researcher (sometimes 'narrowly science-based' (14), or for a doctor sitting on an ethics committee pronouncing on such research, to see the contemplated project as one definable type within a classificatory system. This paper presents such an expanded classification of different types of clinical research which may involve children. It develops the strengths and weaknesses and the specific constraints of each type, from the ethical perspective of the child patient.

\section{Research aimed at the prevention of ill health}

The most logical of all research is that aimed at the prevention of ill health. In adult medicine, cost-benefit ratios of such research may be high due to the increasing costs of high technology (15). By contrast, in the paediatric sphere, most cost-benefit studies of such things as vaccination programmes, genetic counselling clinics, and neonatal intensive care facilities (16) have shown that the outcome is relatively beneficial. Any research endeavour which will reduce the occupancy of child hospital beds will be ultimately cost-effective (17). The underlying axiom of preventitive research in the paediatric domain is that if a life can be saved or disability prevented then the benefits are there to accrue over a future lifetime of perhaps seven decades. 'Prevention research' . . . 'will benefit ourselves and our children . . . in the year 2000' (18). 
Ethical problems relating to individual child subjects are not uncommon in preventive research, decisions about vaccination of children being one example. Simple data collection may be distressing for parents, particularly if high quality detailed data has to be obtained personally. The collection of such data may involve considerable potential interference with parents of deceased or disabled children. There is a potential problem here that in almost all cases it is not the specific individual child or his family who will benefit, but the more global population of his successors in the future. To this end, it is important that a researcher be aware that the ends may not justify the means in this type of research. One has encountered examples of this in studies aimed at the prevention of childhood drowning accidents. To acquire data of acceptable quality concerning the causes of drowning in early childhood (19), it has been necessary to talk with parents, relatives, and neighbours who have lost a child in accidental drowning situations. This is obviously distressing (20), and potentially insensitive. One has to go to great lengths under these circumstances not to cause further distress, however worthy might be the long-term potential benefits of such research. It is important to stress that information gained about children and families in the context of a preventive research programme, is privileged information; and as such, it is subject to the same ethical constraints of confidentiality and accountability normally applicable in the case of an adult clinical patient (21).

Preventive research has already identified what would be undoubtedly effective stratagems to reduce the more prevalent factors causing serious morbidity and mortality in childhood. This applies particularly to factors known to cause mortality in children in the Third World (22), to reduction in the incidence of chromosome abnormalities (23) and to reduction of trauma in childhood (24). Research is now needed to determine how such knowledge can be carried forward into society to effect a reduction in the number of children who are killed, or disabled. There are two required levels of research in the preventive area in this context. The first and perhaps more superficial, should be directed towards facilitating the way in which a target population (for example children or their parents) actually receives the preventive message. The second more fundamental question however, is whether such 'prevention messages' can be better translated into practice, namely to achieve an absolute reduction of sick and injured children.

Prospective preventive research, in its data collection phase, has a danger of altering the parameters being observed - the Hawthorn effect. This phenomenon may lead to ethical dilemmas of whether or not to offer therapeutic intervention. Examples of this research dilemma are known in the field of prospective studies to predict child abuse (7).

Perhaps the major ethical dilemma in funding paediatric research, is the vexed question of whether or not the balance of current paediatric research is still $\stackrel{\frac{m}{5}}{\stackrel{2}{*}}$ under-favouring preventive investigations.

\section{Curative research}

Research involving children who have fatal or gravely debilitating diseases usually has an air of immediacy about it. In such situations the child himself has everything to gain. In this type of situation, there may be an acceptable philosophy that (provided there is no discomfort, and that informed consent is given by parents) the research should be intensive, and pushed to the ethical limit. This in turn has engendered several current ethical dilemmas, one example of which relates to research frontiers in the attempted salvage of neonates at the extreme margin of human viability (25).

The risks of therapeutic research involving children have been ranked (4); absolute risks are probably disease-specific, but remain unknown. One United States study of adult patients showed that some 2.7 per cent of patients sustained more than a trivial injury as participants in therapeutic research (12). Assessment of risks involves not only the degree of potential physical harm, but also an assessment of the psychological sequelae suffered by a person who is harmed (8).

In the case of children afflicted with chronic illnesses, any research endeavour is almost always greeted with gratitude by parents. In this type of case, even although no positive results might ensue, the mere fact that research is continuing, in itself forms a valuable function. It behoves the researcher under these conditions to be doubly aware that unless parents are truly informed about the likely benefits of such research, they may allow their child to be subjected to intervention which has no real potential for helping that child individually, and may have the potential of causing significant distress or discomfort. Children under these circumstances are specially vulnerable (26), but if they have reached the age of reason may well wish to take part in such research which is ostensibly 'curative', but which may entail certain discomfort and perhaps some physical risk. In deciding on the degree of research boldness that is acceptable, one approach is to try and resort to the relative objectivity (or reduced subjectivity) of clinical decision analysis $(27,28)$, and put oneself totally in the child's place.

\section{Research to alleviate symptoms}

This is probably the commonest type of clinical research undertaken in the realm of sick children Themes include those aimed at improving or hastening convalescence, those aimed at reducing secondary effects or complications of primary illness or injury, and those which endeavour to improve the quality of life of sick children. Drug trials, the commonest type of research in adults (1), fall most commonly into this class of research. Properly conducted research with 
these objectives is obviously potentially good, humanitarian and to be encouraged. One example here is the approach to improve the nutritional status of children with cystic fibrosis (29). Other examples might be (a) studies to improve the survival time of children with malignant diseases, and (b) studies to develop better supporting facilities for the physically disabled. Although this type of research can produce immediate short-term gains, this type of clinical investigation has traditionally the greatest failings from the point of view of scientific truth. Placebo effects abound in this area of endeavour. It is unethical to pursue bad research. Unethical, in the author's view both on deontological and on consequentialist views of ethics (30).

Special attention is needed in this area to produce an impeccable methodological approach, and almost always a formal null-hypothesis foundation is required. Special efforts are required to reduce the inadvertent selection of biased cases to a minimum, considerable efforts are required to provide matched controls, and the ever present danger of claiming positive results when all that is happening is a normal regression towards the mean, are ever-present dangers. (This latter is a common pitfall in clinical research. Many chronic diseases naturally wax and wane in severity. If one tries a new method of treatment, and uses only cases in relapse or in a 'bad patch', then many will improve spontaneously as part of the integral waxing and waning cycle. With such selection of cases, many new treatments being studied seem to have a beneficial effect. Once such claims are made in these circumstances it is ethically difficult ever to conduct a proper controlled trial.)

In both the 'symptomatic research' and 'curative research' fields, it is desirable that proper control trials be undertaken early in the history of the new idea, or in the development of a new concept. The reason for this is that if a new endeavour is trialled, and seems to work, there is always grave disquiet on ethical grounds whether one can withhold such putatively-effective treatment from needy children. The history of paediatrics is replete with examples of practices which once introduced, even although their efficacy is doubtful, cannot really ethically be withdrawn until a long passage of time ultimately proves their inefficiency. When society generally, and paediatricians specifically, believe that a certain form of treatment may hold hope, it is almost impossible to withhold such treatment from an individual child who suffers from the disease in question. The best ethical approach, when a new idea arises, is to conduct a short and well planned pilot study, and then to go straight to a detailed controlled study which will resolve the question.

\section{Research to establish norms and baselines}

This is a very important type of research which involves children, as the core feature of childhood as a biological state is its constant change. Such change, both in terms of physical growth, and intellectual and social development, means that norms and baselines have to be established for each age and sex, and ideally also for all other factors known to influence development. The influence of socio-economic status on children's health is enormous (31). Thus this type of descriptive research to define normal states of the well child (for example percentile charts) has to be undertaken separately in children of different race, genetic background, socio-economic status, and country of residence. In this area, there remains an unfulfilled need for percentile charts of physiological and performance variables throughout childhood, as an extension to simple height, weight and head circumference tables. Recently developed norms for handgrip strength are an example here (32).

There are obvious limits to this type of research, just as in the area of 'curiosity research'. In the case of children there has to be a no-harm criterion, the concept of harm again meaning not only physical pain or danger, but intrusion into a child's life in ways and degrees which are less optimal for his normal development than might be other recreational or learning activities.

In this type of research, it is sometimes easier to achieve motivation for participation in non-invasive research involving children, than it is in corresponding groups of adults. Children tend not to see the need for personal gain if they are involved in a research enterprise. Most children, also, do not see their participation in this type of research as in any way intrusive, and if the testing scenario is made relaxed and enjoyable, participation in such a research project can be very meaningful, and worthwhile for the participants as well as for the research itself. This subjective attitude of child subjects, however pleasurable, does not of course justify undertaking research, which must be justifiable on independent grounds. One specialised area where this absence of normative data is particularly pertinent is in the field of antenatal diagnosis of congenital abnormalities. Anthropometric data is now well established for most indices which can be measured in normal fetuses. However, the patterns of such development for fetuses with genetic diseases and with congenital abnormalities are generally unknown. These data are urgently required, and will take several decades still to generate. Such research is not intellectually taxing, but requires an experienced objective approach to ensure that bias is not introduced.

A special problem relating to this type of 'baseline research', if sick children are being studied, is the use of normal children to establish the normal range of the variable under consideration. This is an almost insuperable problem and there is often no alternative which is both efficient on the one hand, and ethical on the other. An example of this difficulty might be the execution of a study to rank the different radiological features which indicate raised intra-cranial pressure in children with such things as cerebral tumours. It is 
simple to collect case series of such children with cerebral tumours and to study the radiological changes in their skull $\mathrm{x}$-rays or CAT scans which are taken for valid clinical reasons. However, how does one collect a series of matched controls of normal children of the same age and sex? Obviously not by $x$-raying normal children's skulls. One has to compromise under these circumstances, and gradually accumulate a series of skull radiographs taken for other purposes, such as acute head injury, or collect a series of autopsy skull radiographs of children dying from other causes. Even then, there is always some doubt about the selected nature of such 'control' series, and in particular there is always concern about the validity of quantitative parameters drawn from them.

One other type of basic research which involves in a sense the simple description of syndromes, or diseases, is nosological (ie disease-classificatory) research aimed at the definition of syndrome boundaries. This type of research is not directly curative, nor would it help the symptoms or complications of disease in childhood, but it is important from the point of view of genetic counselling, and from the clinical aspect of being able to give an accurate prognosis in the case of individual children afflicted by the disease in question. Two examples with current clinical topicality are the development of differential diagnosis (with its underlying nosological themes) in the field of neuromuscular disease in childhood (33), and the finer and finer prognostic tuning relating to survival in acute leukaemia, following cytogenetic, immunological and histochemical classification. Historically, approaches to the understanding of many diseases in childhood have centred on nosological research endeavours, a topic formalised in the great debates between 'lumpers' and 'splitters', terms first coined by McKusick in 1969 (34). Such approaches have endeavoured to determine whether clinical syndromes are in reality groups of overlapping (but different) diseases, or comprise single entities with broad clinical spectra. Although the answers to such questions involve intrusion into families with affected children, such answers form the sole basis on which accurate prognoses can be given in individual cases.

\section{Curiosity research}

Research 'simply to discover' is research motivated by curiosity alone. In research of this type, there is no hypothesis to be tested, and no data to be generated to be used as baselines. The 'needs of science' (13) in this case are very low. In many other types of clinical research procedure involving children, it is difficult to answer the question 'Does the procedure benefit the patient, or promote the researcher's interests?' (35). In curiosity research, there is no subtle dilemma - the project benefits the researcher only, in the short term.

Curiosity research is not looked upon favourably in the fashion of modern research trends, and is not commonly funded by bodies dispensing funds for $\frac{\Pi}{F}$ which competition is vigorous. This is in many ways unfortunate, as a review of the historical advances in the fields of medicine and biology, and in the basic $\overrightarrow{\vec{F}}$ sciences, reveals that curiosity research plays a $\frac{7}{0}$ fundamental role in the advancement of knowledge $\frac{C}{0}$ both basic and applied (36). There is a distinction $\overline{\frac{D}{5}}$ between curiosity research and non-therapeutic $\widetilde{\Phi}$ research. Non-therapeutic research uses models of $\varrho$

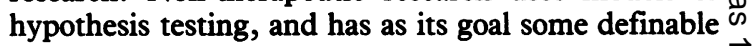
clinical objective, albeit one which will not help the $\overrightarrow{0}$ individual research subject directly. The human costs $\overrightarrow{\vec{C}}$ of non-therapeutic and 'curiosity' research in current $\stackrel{\sigma}{\circ}$ practice are low, but are measurable (12), at least in $\widehat{\overline{3}}$ adults.

One appreciates that any project which is $\vec{\omega}$ fundamentally of this type (primary curiosity) can be cloaked with an hypothesis to be tested, in a contrived $\mathcal{o}^{\circ}$ way. It is one function of institutional review boards, ? or hospital ethics committees, to determine whether a $\rightarrow$ project under review falls into this category or not. In 3 the special case of research involving children, the researcher motivated simply by curiosity (or the desire $\mathcal{O}$ to advance knowledge concerning children, for its own $\vec{\bullet}$ sake) has several special constraints (2). Children are specially susceptible to a number of agents (such as radiation effects) when compared with adults (37). Not only must there be no risk of unpleasantness involved in any such endeavours, but the study must not displace other beneficial endeavours such as time at $\frac{}{\varnothing}$ school. Interestingly, such research can have a direct $\cong$ benefit, by example. If a child (a subject) experiences $\overrightarrow{\overrightarrow{0}}$ the interests and endeavours of a research project, this may become a model for the wider world of curiosity and of its place in self-fulfillment and learning, so essential for all individuals in society.

\section{Conclusion}

Ethical overtones in medicine are, inter alia, based on two special themes - (a) a genuine belief that what one is doing is helpful to the patient, and (b) primum non 음 nocere. Ethical issues however are more complex in practice in 1985 (38), and in the context of research involving children both principles cannot be absolute. Hopefully, the older approach that discussion about ${ }^{-N}$ ethical argument is not fruitful (everyone's ethical o claims are as good as another's) has now passed (39). N

Labelling specific issues of paediatric research with $\bar{r}$ their ethical implications removes them from theo category of being exclusively medical (3). The child is $\Phi$ the consumer in the paediatric research industry. $\stackrel{\infty}{+}$ Strengthening of consumer rights need not necessarily imply any future reduction in paediatric research, but $\frac{\text { }}{\mathbb{D}}$

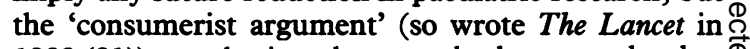
1983 (21)), emphasises tht not only the researcher but $\stackrel{\mathbb{Q}}{\varrho}$ also the paediatric patient are contributing to produce something new. This applies also to non-therapeutic research, which can be further sub-divided into the themes of preventive research, research to establish norms and baselines, and curiosity research. Sick $\stackrel{\bar{\partial}}{\vec{P}}$ 
children, and potentially sick children of the future, need a research climate in which each of these five ongoing investigational themes are further encouraged.

Fohn Pearn MD PhD is Head of the Department of Child Health at the Royal Children's Hospital, Herston, Brisbane, Queensland, Australia and Chairman of the Ethics Committee of the Royal Children's Hospital, Brisbane.

\section{References}

(1) Allen P A, Waters W E. Development of an ethical committee and its effects on research design. Lancet 1982; 1: 1233-1236.

(2) Pearn J H. The child and clinical research. Lancet 1984; 2: $510-512$.

(3) Abram M B, Wolf S M. Public involvement in medical ethics. New England journal of medicine 1984; 310: 627632.

(4) Janofsky J, Starfield B. Assessment of risk in research on children. Fournal of pediatrics 1981 ; 98: 842-846.

(5) Fost N. Risks of research on children. Fournal of pediatrics 1982; 100: 166-167.

(6) Fost N. Parental control over children. Fournal of pediatrics 1983; 103: 571-572.

(7) Landwirth J. Ethical aspects of studying child abuse. Fournal of pediatrics 1983; 102: 322.

(8) Leikin S L. Risks of research on children. Fournal of pediatrics 1982; 100: 166.

(9) Phibbs R H. Ethical issues in neonatology. The TurnerGibson oration, 1981. Australian paediatric journal 1981; 17: 247-249.

(10) National Health and Medical Research Council Working Party on Ethics in Medical Research. Ethics in medical research. Canberra: Australian Government Public Service, 1983.

(11) Australian College of Paediatrics Council. Report on the ethics of research in children. Australian paediatric journal 1981; 17: 162.

(12) Cardon P V, Dommel F W, Trumble R R. Injuries to research subjects. New England journal of medicine 1976; 295: 650-654.

(13) Lowe C U, Alexander D, Mishkin B. Non-therapeutic research on children: an ethical dilemma. Fournal of pediatrics 1974; 84: 468-473.

(14) Anonymous. Medical ethics. [editorial]. Lancet 1982; 1: 260-261.

(15) Golding A M B, Tosey D. The cost of high-technology medicine. Lancet 1980; 2: 195-197.

(16) Banta H D, Thacker S B. Assessing the costs and benefits of electronic fetal monitoring. Obstetric and gynaecological survey 1979; 34: 627-630.

(17) Smith D M, Roberts S D, Gross T L. Health care costs of medical patients at an urban center. Fournal of community health $1981 ; 7: 21-32$.

(18) Funder J. The budget and medical research. Medical journal of Australia 1984; 141: 692-693.
(19) Pearn J H, Nixon J. An analysis of the causes of freshwater immersion accidents involving children. Accident analysis preview 1979; 11: 173-178.

(20) Nixon J, Pearn J. Emotional sequelae of parents and sibs following the drowning or near-drowning of a child. Australia and New Zealand journal of psychiatry 1977; 11: 265-268.

(21) Anonymous. Confidentiality and accountability [editorial]. Lancet 1983; 1: 277-278.

(22) UNICEF. The state of the world's children. Geneva: UNICEF (UN), 1984

(23) Bell J A, Pearn J H, Bowling F, et al. Factors influencing referrals for prenatal cytogenetic diagnosis. Australia and New Zealand journal of obstetrics and gynaecology (in press).

(24) Baker S P. Prevention of childhood injuries. Medical journal of Australia 1980; 1: 466-470.

(25) Campbell A G M. Which infants should not receive intensive care? Archives of disabled children 1982; 57: 569-571.

(26) Takanashi R. Childhood as a social issue. Historical roots of contemporary child advocacy movements. Fournal of social issues $1978 ; 34: 8-28$.

(27) Brett A S. Hidden ethical issues in clinical decision analysis. New England journal of medicine 1981; 305: 1150-1152.

(28) Bourke D L. Ethical issues in decision analysis. New England journal of medicine 1982; 306: 613.

(29) Miller M, Ward L, Thomas B J et al. Altered body composition and muscle protein degradation in nutritionally growth retarded children with cystic fibrosis. American journal of clinical nutrition 1982;36: 429-433.

(30) Gillon R. An introduction to philosophical medical ethics: the Arthur case. British medical journal 1985; 290: 1117-1119.

(31) Nixon J, Pearn J. Norms for the social class distribution Medical journal of Australia 1980; 2: 271-273.

(32) Newman D, Newman J, Pearn J. Norms for handgrip strength. Archives of disabled children 1984; 59: 453-459.

(33) Pearn J. The discovery of neuronopathy and neuropathy as a cause of progressive paralysis in childhood. Fournal of neurological science 1984; 64: 99-107.

(34) McKusick V A. On lumpers and splitters or the nosology of genetic disease. In: Bergsma D, ed. Proceedings of the first conference of clinical delineation of birth defects, Part I, Vol 23. London: National Foundation March of Dimes, 1969.

(35) Jonsen A R. Watching the doctor. New England journal of medicine 1983; 308: 1531-1535.

(36) d'Abro A. The methodology of science. In: d'Abro A. The evolution of scientific thought. New York: Dover Publications, 1950: 343.

(37) Finberg L, Scriver C R. Special susceptibility of children to radiation effects. Pediatrics 1983; 72: 890.

(38) Stone A L. Law's influence on medicine and medical ethics. New England journal of medicine 1985; 312: 309 312.

(39) Gillon R. It's all too subjective: scepticism about the possibility or use of philosophical medical ethics. British medical journal 1985; 290: 1574-1575. 